\title{
Pancreatic Duct Stenosis, CTCAE
}

National Cancer Institute

\section{Source}

National Cancer Institute. Pancreatic Duct Stenosis, CT CAE. NCI Thesaurus. Code C143730.

A disorder characterized by a narrowing of the lumen of the pancreatic duct. 